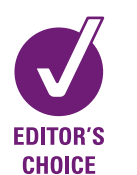

CHOICE

\title{
Novel non-occlusive temporary endoluminal neck protection device to assist in the treatment of aneurysms in a canine model
}

\author{
Raymond D Turner, ${ }^{1}$ M Imran Chaudry, ${ }^{2}$ Aquilla S Turk ${ }^{2}$
}

\begin{abstract}
${ }^{1}$ Stroke and Cerebrovascular Center, Department of Neurosciences, Medical University of South Carolina, Charleston, South Carolina, USA ${ }^{2}$ Stroke and Cerebrovascular Center, Department of Radiology, Medical University of South Carolina, Charleston, South Carolina, USA
\end{abstract}

\section{Correspondence to} Raymond D Turner, Stroke and Cerebrovascular Center Department of Neurosciences, Medical University of South Carolina, 96 Jonathan Lucas Street, Suite 428 CSB, Charleston, SC 29466, USA; turnerrd@musc.edu

Received 29 September 2010 Revised 24 November 2010 Accepted 30 November 2010 Published Online First 25 January 2011

\begin{abstract}
Introduction Current models for the endovascular treatment of aneurysms often require the use of adjuvant devices. Balloon-assisted techniques require temporary occlusion of the parent vessel. Intravascular stents require the use of antiplatelet medication. A novel device that achieves temporary neck protection without parent vessel occlusion or antiplatelet medication is examined in a canine model.
\end{abstract}

Method 20 sidewall wide-necked aneurysms were created in 10 canines, one in each carotid artery, using a vein graft technique, and allowed to mature. In each canine, one aneurysm was catheterized with

a microcatheter while the neck protection device (NPD) was unsheathed across the neck of the aneurysm and the aneurysm was coiled. The second aneurysm in each canine was coiled without an adjunctive device. The NPD was assessed for coil herniation, coil entrapment within the device, deployability and retrievability. Five animals were killed immediately and five were killed at 28 days and the carotid artery was explanted and sent for necropsy to assess for injury to the endothelium.

Results All aneurysms were successfully treated with no adverse events. The NPD was well visualized and unsheathed and resheathed successfully in all cases. There was no coil entrapment within the device, and there were no cases of coil herniation. There was no evidence of endothelial injury or dissection.

Conclusion The device was technically successful in all devices and further development of this device may be of benefit in endovascular treatment of aneurysms.

\section{INTRODUCTION}

Current endovascular treatment of aneurysms often requires the use of an adjuvant device to prevent coil herniation into the parent vessel during endovascular obliteration of an aneurysm. The balloon-assisted technique (BAT) utilizes intermittent occlusion of the parent vessel while a second microcatheter is deploying coils into the aneurysm. ${ }^{1}$ It is common to inflate and deflate the balloon several times during the treatment, with inflation times varying based upon aneurysm location, collateral vessels and surgeon preference. Patients are heparinized to prevent thrombus formation during occlusion.

Intravascular microstents can also be used to assist in the treatment of aneurysms. ${ }^{2-4}$ They can be deployed prior to catheterization, which requires microcatheterization of the aneurysm through the stent, or they can be deployed after aneurysm catheterization, effectively jailing the microcatheter into the aneurysm. The former technique can be susceptible to stent shift or migration during the placement of the microcatheter through the stent, while in the latter technique, the jailed microcatheter creates a potential space for coil herniation along the catheter due to incomplete opposition of the stent-parent artery wall interface along the microcatheter. Both techniques require the use of antiplatelet medication to prevent thrombus formation and have been shown to carry a high risk of major morbidity and mortality associated with its use in the setting of ruptured aneurysms. ${ }^{56}$ There is also a small risk of stenosis, which can develop within the stent during the vessel's healing process.

A novel temporary aneurysm neck bridge system (TANBS) (Mindframe Inc., Lake Forest, California, USA), which achieves temporary neck protection without parent vessel occlusion or long-term antiplatelet medication is examined in a canine model. The aim of this project is to assess the device as a potential adjunctive device for coil embolization and evaluate the device for safety through histological evaluation of the catheterized vasculature.

\section{METHODS}

This study was performed under a protocol approved by the Institutional Animal Care Committee at the Medical University of South Carolina. Analgesia and anesthesia were administered according to the standard operating procedure of the university.

After induction of anesthesia, 20 sidewall aneurysms were created in 10 canines, one in each cervical carotid artery, using a vein graft technique previously described, ${ }^{8}$ and allowed to mature for 2 weeks. Under anesthesia, the common femoral artery was accessed and a $6 \mathrm{~F}$ guide catheter was placed into the carotid artery. All aneurysms were coiled using currently available, FDA-approved platinum coils from a single vendor. In each canine, one aneurysm was catheterized with a microcatheter and coiled to occlusion while the TANBS was unsheathed across the neck of the aneurysm. The second aneurysm, if patent, was coiled to occlusion without the use of an adjunctive device. The TANBS was assessed for coil herniation, coil entrapment within the device, radio-opacity, movement through the device, trackability, movement of the guidewire through the system, TANBS deployability, TANBS resheathing and TANBS retrievability using the following grading scale: 1 , unsatisfactory; 2 , difficult; 3 , adequate; 4 , good; 5 , excellent. Five animals were killed immediately, and five survived 28 days then were killed, and the carotid arteries were explanted and sent for blinded necropsy at an independent facility to assess for evidence of injury to the endothelium. 


\section{Device}

The TANBS is a self-expanding, closed-cell nitinol mesh engineered from a laser-cut hypotube in $23-\mathrm{mm}$ and $32-\mathrm{mm}$ lengths, $5 \mathrm{~mm}$ in diameter (figure 1). The diameter of the device fully expanded is $5 \mathrm{~mm}$ and it has a cell size that is smaller than that of the Solitaire stent (ev3; Plymouth, Minnesota, USA) and similar in cell size to the Neuroform (Boston Scientific, Natick, Massachusetts, USA) and Enterprise (Cordis Neurovascular, Miami Lakes, Florida, USA) stents (figure 2). It is mounted on a pusher created via a hypotube to allow for an over-the-wire design such that the device can be navigated into position over a 0.010 inch $=$ 0.254 millimeters microwire. The inner lumen of the TANBS and pusher is 0.0163 inch $=0.41402$ millimeters allowing for a luminal capacity around the 0.010 -inch microwire for intraarterial infusion of saline or medication. The TANBS has an outer diameter of 0.0225 inch $=0.5715$ millimeters. The entire system is designed to be delivered through all commercially available 0.027 inch $=0.6858$ millimeters microcatheters. The device is deployed by positioning the device across the intended location and withdrawing the catheter, which unsheathes the device. Resheathing is performed by advancing the catheter over the device while it is fixed in position. The outward radial force varies based on the size of the target vessel; however, it is similar to the Solitaire stent, is consistently greater than the Enterprise stent and consistently lower than the Neuroform stent (figure 3).

\section{Histology}

The specimens were explanted and evaluated at an independent pathology laboratory for necropsy by a veterinarian pathologist who was blinded to which aneurysm and attached parent artery were catheterized with the TANBS and coiled and which specimens were in the control arm. All specimens were fixed in formalin, then embedded in methyl methacrylate, polished, sectioned and stained with $\mathrm{H} \& \mathrm{E}$. The parent arteries were examined for evidence of endothelial injury. The aneurysms were graded for the presence of fibroblasts and inflammation using the following scale: 1 , minimal; 2 , mild; 3 , moderate; 4, moderate-severe; 5 , severe.

\section{RESULTS}

There were 17 aneurysms present for coiling out of the 20 aneurysms originally created. Three aneurysms thrombosed during the 2-week healing period and were occluded on angiography at the time of intended coiling. All 17 patent aneurysms

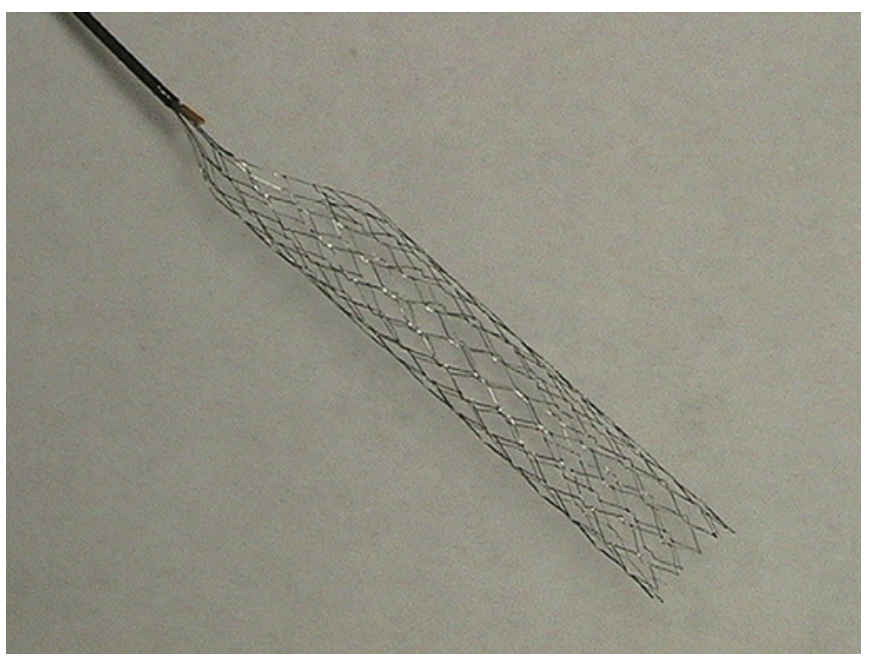

Figure 1 Temporary aneurysm neck bridge system, $5 \mathrm{~mm} \times 32 \mathrm{~mm}$.

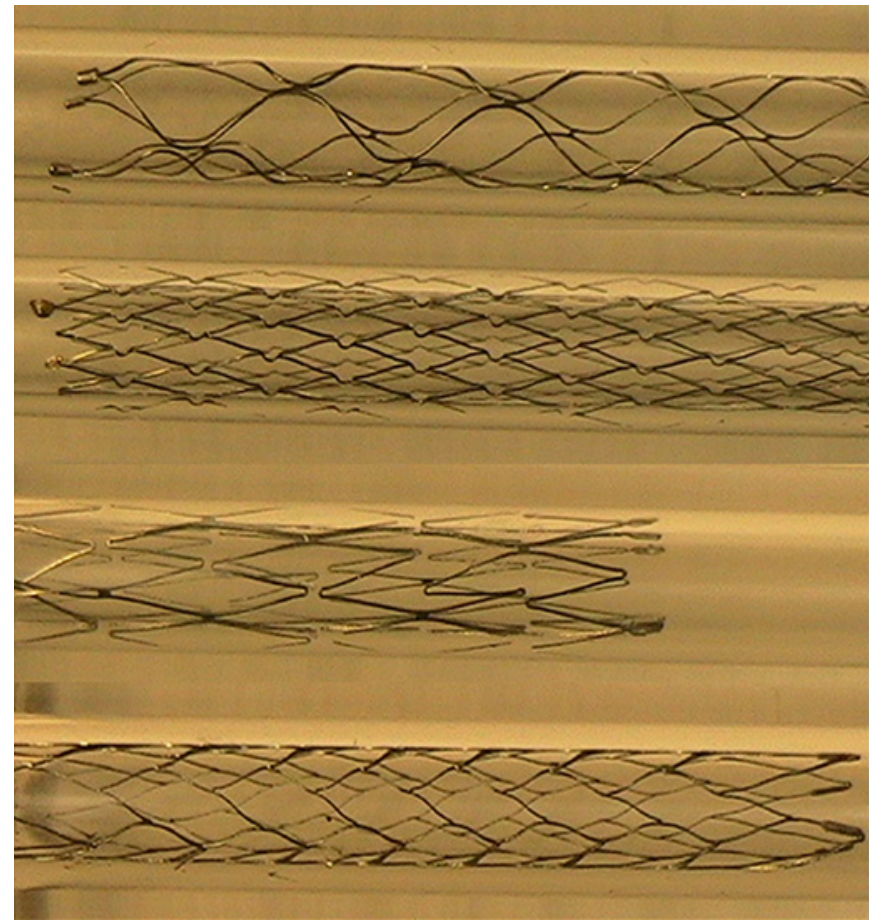

Figure 2 Glass tubes $(3 \mathrm{~mm})$ with the Solitaire $(\mathrm{eV} 3)$, temporary aneurysm neck bridge system (TANBS), Neuroform and Enterprise devices demonstrating cell size and shape.

were successfully coiled to complete occlusion and explanted at their respective time-points. Ten of the aneurysms, one in each of the 10 canines, were coiled to occlusion while the TANBS device was deployed across the neck of the aneurysm. The seven remaining aneurysms located on the contralateral carotid artery were coiled to occlusion without the assistance of any neck protection devices. Immediate occlusion and a dense coil mass were achieved in all cases.
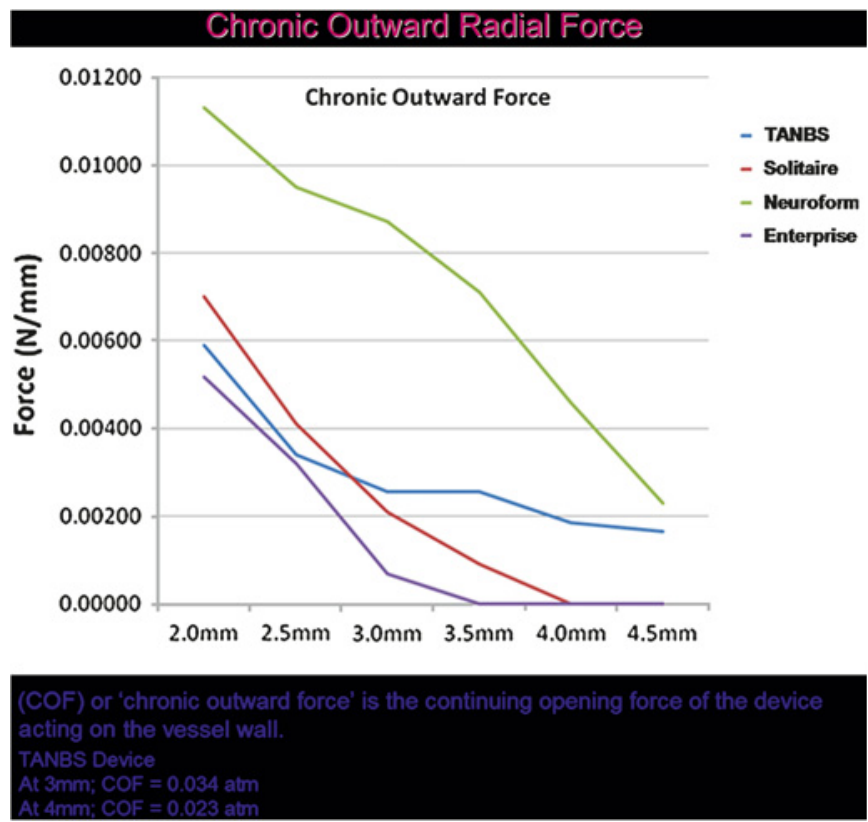

Figure 3 Chronic outward radial force as increasing diameters of deployment. Note that the temporary aneurysm neck bridge system (TANBS) has a chronic outward radial force that is consistently greater than that of Enterprise, and less than that of Neuroform. 
The TANBS was successfully moved through the delivery catheter with an excellent grade $($ score $=5)$ in all cases. The device was navigated into position across the aneurysm neck, deployed and resheathed without adverse events in all 10 aneurysms, receiving an excellent grade $($ score $=5)$ in all canines. The device was successfully removed in all cases. The 0.010 -inch microwire moved well through the TANBS and received an excellent grade in all cases. Coils were successfully placed into the aneurysms without evidence of coil herniation around the device or through the interstices of the device. The device was resheathed following completion of the coiling. There were no instances of coil entrapment within the device or coil mass shifting as a result of the device being removed. The ability to infuse through the TANBS was not assessed in this study.

The radio-opacity of the device was adequate using GE/OEC 9800 C-Arm. The device had excellent visualization while constrained in the catheter as well as when deployed in the parent artery. Angiography did not reveal evidence of vasospasm or vessel dissection following the removal of the device. There was no change in the aneurysm occlusion result following the removal of the device. During device resheathing there was no evidence of interaction with the deployed coils as evidenced by movement of a coil loop or change in configuration of the coil mass (figure 4).

Fibroblasts were associated with all but two aneurysms (chronic arm, non-patent and not coiled). The third uncoiled, non-patent aneurysm (acute arm) was associated with minimal fibroblasts. Four coiled aneurysms had mild fibroblasts, two were in the TANBS group and two were coiled without assistance. The largest aneurysm was associated with the greatest degree of fibrosis (table 1).

In the three aneurysms that were not patent for coiling, there was no inflammatory response. Three specimens in two canines were associated with severe inflammation, both carotids in one canine and the control carotid in a second animal. A third animal had a moderate-severe inflammatory response in the TANBS group. All other aneurysms were associated with minimal or

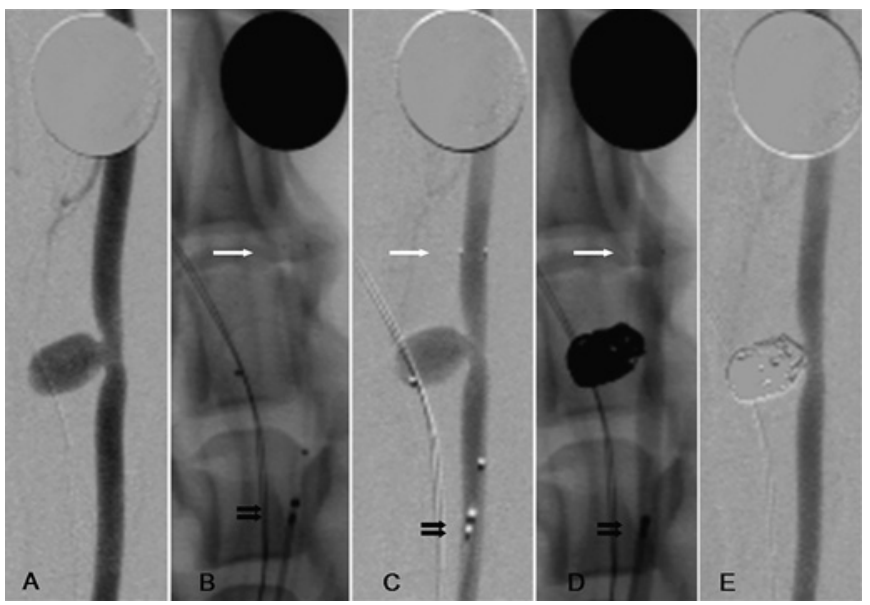

Figure 4 Angiograms of the carotid artery with sidewall vein pouch aneurysm. A dime is present at the top of every picture for size reference. (A) Aneurysm prior to treatment. (B) (Native) and (C) (DSA): demonstrating that the temporary aneurysm neck bridge system (TANBS) is deployed across the aneurysm; white arrow, distal marker; double black arrow, proximal marker. Note the two markers present demonstrating that the aneurysm is catheterized. (D) After coiling with microcatheter removed and TANBS present (arrows). (E) Final DSA with TANBS removed demonstrating occlusion of the aneurysm and no evidence of vasospasm or intimal flap/endothelial injury. mild inflammatory response. Overall, fibrosis was greatest in the chronic (28-day) group, while inflammation was most prominent in the acute group. The largest aneurysm was partially recanalized at 28 days, occurring in the TANBS group. Three aneurysms were associated with suture-related granulomas. There were no cases of parent vessel endothelial injury, perforation or intramural dissection.

\section{DISCUSSION}

Aneurysm coiling with currently available devices is commonplace. However, aneurysm coiling often requires the use of an adjunctive device, most often a balloon or microstent. These devices have the potential to increase the complexity of the case. The BAT uses temporary parent artery occlusion at the site of the aneurysm to prevent coil herniation into the parent artery. ${ }^{1}$ The balloon delivery systems are often navigated into position within the same guide catheter, which creates competitive forces between the balloon catheter and the microcatheter due to friction and differences in catheter loading, which may result in the build-up of potential energy within one of the catheter systems. Ovalization of a guide catheter due to tortuosity can potentiate some of these issues. In the canine aneurysm model, there is very little tortuosity and as a result, there was little interaction between the TANBS device and the microcatheter; however, this is more likely to be related to the animal model design. It is anticipated that in a model designed with tortuosity that more closely mimicks the human, there will likely be catheter interactions similar to those seen with balloon remodeling techniques.

Stent-assisted coiling can be accomplished as a stand-alone device, or in addition to the BAT, either after the coiling has been completed or as a balloon in stent technique. Stenting as a standalone adjunctive device can be accomplished prior to aneurysm microcatheterization, requiring the microcatheter to navigate through the stent, or after microcatheterization, which effectively 'jails' the microcatheter. In the former scenario, the stent has the potential to shift or move, while in the latter, the catheter placement between the stent and the parent vessel creates a small space where the stent is not abutting the artery wall, creating a space for coil loops to herniate.

Both adjunctive devices have the potential to create thromboembolic events, thus judicious management of anticoagulation is required to prevent complication. Although BATs do not require long-term antiplatelet treatment after the procedure, stent placement does. ${ }^{2}$ Further, the long-term effects of stents implanted in the cerebral vessels remain unknown.

The novel device used in this experiment is designed to incorporate the positive attributes of existing adjuvant devices without the known limitations. The TANBS allows temporary, non-occlusive protection of the parent artery during coil embolization of aneurysms. Unlike implantable microstents, this device does not require long-term antiplatelet therapy because it is removed at the end of the procedure. The TANBS appears to provide a neck protection profile similar to what we expect with stenting. Its potential advantage over BATs of aneurysm coiling is that it provides similar protection of the parent artery without temporary occlusion of the vessel. However, in the setting of an intraprocedural aneurysm rupture, balloons provide definitive and immediate occlusion to limit hemorrhaging, a level of protection that TANBS and stents cannot provide.

The device performed well in all 10 cases and appeared to provide similar neck protection to that of a conventional 
Table 1 Necropsy results at acute and 28 days detailing the degree of fibrosis and inflammation present in the specimens

\begin{tabular}{|c|c|c|c|c|c|}
\hline Animal number & Tissue & Fibroblasts & Inflammation & Other & Comments \\
\hline \multicolumn{6}{|l|}{ Acute } \\
\hline CYFAM1 & Left carotid & 4 & 2 & NSF & Mononuclear inflammation with fewer neutrophils \\
\hline CYFAM1 & Right carotid & 2 & 1 & NSF & Mononuclear inflammation with multifocal discrete granulomas \\
\hline CYHAMN & Left carotid & 4 & 5 & FM & Inflammation is mostly pyogranulomatous \\
\hline CYHAMN & Right carotid & 3 & 5 & $\mathbf{F M}$ & Inflammation is mostly pyogranulomatous \\
\hline CYHBBR & Left carotid & 3 & 1 & NSF & Very few neutrophils with hemosiderin-laden macrophages \\
\hline CYHBBR & Right carotid & 1 & 0 & Granulomas & There are several peripheral suture granulomas \\
\hline CYF BEF & Left carotid & 4 & 4 & FM & The inflammation is mostly pyogranulomatous \\
\hline CYF BEF & Right carotid & 3 & 2 & NSF & Inflammation is mostly mononuclear with fewer neutrophils \\
\hline CYFAY5 & Left carotid & 2 & 1 & NSF & Mononuclear inflammation with fewer neutrophils \\
\hline CYFAY5 & Right carotid & 2 & 1 & NSF & Mononuclear with hemosiderophages and fewer granulomas \\
\hline \multicolumn{6}{|l|}{ Chronic: 28 days } \\
\hline CYHAPW & Left carotid & 5 & 1 & RT & $\mathrm{RT}=$ recanalized thrombus \\
\hline CYHAPW & Right carotid & 4 & 2 & NSF & No lumen identified \\
\hline CYGAFP & Left carotid & 5 & 1 & NSF & Very few macrophages and lymphoplasmacytes \\
\hline CYGAFP & Right carotid & 0 & 0 & NSF & \\
\hline CYFASK & Left carotid & 4 & 2 & NSF & Mostly mononuclear inflammation with suture granulomas \\
\hline CYFASK & Right carotid & 0 & 0 & NSF & \\
\hline CYFBNG & Left carotid & 2 & 1 & NSF & \\
\hline CYFBNG & Right carotid & 4 & 1 & NSF & Mononuclear inflammation \\
\hline CYFBIM & Left carotid & 5 & 2 & FM & Mostly mononuclear inflammation with suture granulomas \\
\hline CYFBIM & Right carotid & 3 & 5 & FM & Pyogranulomatous inflammation \\
\hline
\end{tabular}

1, minimal; 2, mild; 3, moderate; 4, moderately severe; 5 , severe.

FM, foreign material present; NSF, no significant findings.

microstent. There were no instances of coil entrapment or coil mass shifting during the retrieval of this device. There were no observed instances of coil loops herniating into the lumen of the device. Currently available microstents with similar cell sizes can allow for coil loop herniation through the cells of the microstent; an event that is often easily remedied by repositioning of the coil. There was one case of aneurysm recanalization out of the 10 aneurysms treated with the assistance of this device. There were no instances of parent artery vasospasm or endothelial injury seen on angiography during the procedure, which was confirmed on necropsy.

Analysis of the necropsy demonstrates that there was no evidence of endothelial injury related to the navigation of the device into position, its deployment or retrieval as compared with the control side. As anticipated, aneurysm-related inflammation was more common in the acute arm, while fibrosis was more consistent in the chronic group. The inflammation was unusually pronounced in both aneurysms in one canine, and the control aneurysm in a second canine, with the second aneurysm in that canine (TANBS aneurysm) having only mild

\section{Key messages}

Current endovascular treatment of aneurysms often requires the use of an adjuvant device to prevent coil herniation into the parent vessel during endovascular obliteration of an aneurysm. Balloon-assisted techniques and intravascular microstents have benefits and limitations. A novel temporary aneurysm neck bridge system, which achieves temporary neck protection without parent vessel occlusion or long-term antiplatelet medication is examined in a canine model. This device may provide benefits over other currently available devices designed to assist in the treatment of aneurysms. inflammation. Both of these canines were in the acute arm of the study. It is unclear why there was such a discrepancy in the inflammation, but it may be due to variation in the immune response of the animals, the local vascular supply and the degree of tissue disruption that occurred during the creation of the aneurysms 2 weeks previously. The largest of the aneurysms had the greatest degree of fibrosis; however, that was also the aneurysm that recanalized thrombus within the aneurysm sac on necropsy at 28 days.

\section{CONCLUSION}

The TANBS device was technically successful in all cases and provided parent artery protection as was intended. There were no adverse events related to its use. Necropsy demonstrated that there was no evidence of endothelial injury related to the device. This device may provide benefits over other currently available devices designed to assist in the treatment of aneurysms.

Acknowledgments The authors would like to thank Brian Croy and Dan Consigny for their assistance during the study and Adrian Parker for manuscript preparation.

Competing interests None.

Provenance and peer review Not commissioned; externally peer reviewed.

\section{REFERENCES}

1. Malek AM, Halbach W, Phatouros CC, et al. Balloon-assist technique for endovascular coil embolization of geometrically difficult intracranial aneurysms. Neurosurgery 2000;46:1397-406.

2. Fiorella D, Albuquerque FC, Deshmukh VR, et al. Usefulness of the Neuroform stent for the treatment of cerebral aneurysms: results at initial (3-6-mo) follow-up. Neurosurgery 2005;56:1191-201.

3. Fiorella D, Albuquerque FC, Han $\mathrm{P}$, et al. Preliminary experience using the Neuroform stent for the treatment of cerebral aneurysms. Neurosurgery 2004; 54:6-16.

4. Lubicz B, François 0 , Levivier $\mathrm{M}$, et al. Preliminary experience with the enterprise stent for endovascular treatment of complex intracranial aneurysms: potential advantages and limiting characteristics. Neurosurgery 2008;62:1063-9. 
5. Horowitz M, Jovin T. Neuroform Stenting in Acutely Ruptured Aneurysms. Society of Neurolnterventional Surgery, 2009.

6. Tumialán LM, Zhang YJ, Cawley CM, et al. Intracranial hemorrhage associated with stent-assisted coil embolization of cerebral aneurysms: a cautionary report. J Neurosurg 2008;108:1122-9.
7. Fiorella D, Albuquerque FC, Woo H, et al. Neuroform in-stent stenosis: incidence, natural history, and treatment strategies. Neurosurgery 2006;59:34-42.

8. German WJ, Black PW. Experimental production of carotid aneurysms. N Engl J Med 1954;250:104-6.

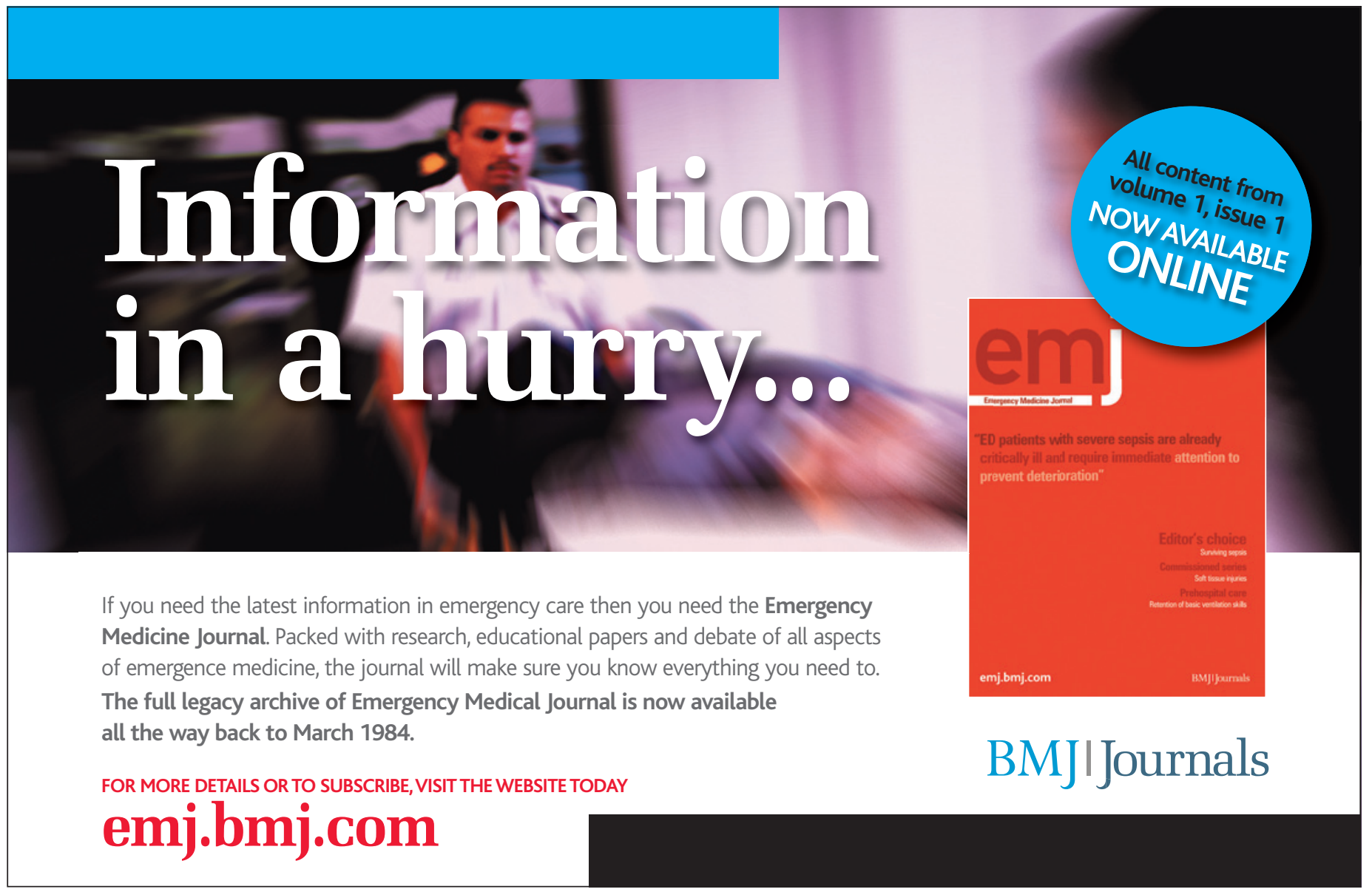

